\title{
VALADEAU Céline, Médecine chez les Yanesha d'Amazonie péruvienne : la traversée par les plantes
}

L'Harmattan, Paris, 2012, 384 p., bibliogr., cartes, ill. et annexes.

\section{Damien Davy}

\section{OpenEdition}

\section{Journals}

\section{Édition électronique}

URL : https://journals.openedition.org/jsa/14713

DOI : 10.4000/jsa. 14713

ISSN : $1957-7842$

\section{Éditeur}

Société des américanistes

Édition imprimée

Date de publication : 31 octobre 2016

Pagination : 213-215

ISSN : 0037-9174

Référence électronique

Damien Davy, " VAladEAu Céline, Médecine chez les Yanesha d'Amazonie péruvienne : la traversée par les plantes », Journal de la Société des américanistes [En ligne], 102-1 | 2016, mis en ligne le 05

décembre 2016, consulté le 04 septembre 2022. URL : http://journals.openedition.org/jsa/14713 ; DOI : https://doi.org/10.4000/jsa. 14713

Ce document a été généré automatiquement le 4 septembre 2022.

Tous droits réservés 


\title{
VALADEAu Céline, Médecine chez les Yanesha d'Amazonie péruvienne : la traversée par les plantes
}

L'Harmattan, Paris, 2012, 384 p., bibliogr., cartes, ill. et annexes.

\author{
Damien Davy
}

\section{RÉFÉRENCE}

VALADEAU Céline, Médecine chez les Yanesha d'Amazonie péruvienne : la traversée par les plantes, L'Harmattan, Paris, 2012, 384 p., bibliogr., cartes, ill. et annexes.

1 Les ouvrages abordant les relations qu'entretient un groupe culturel avec ses plantes médicinales par une approche tant ethnobotanique que d'anthropologie médicale ne sont pas nombreux. Le livre de Céline Valadeau constitue une exception de taille. La traversée par les plantes qu'elle nous propose permet d'approcher la société yanesha (groupe arawak de 7658 âmes vivant en Amazonie péruvienne) par le prisme de leur représentation du monde végétal et de leur étiologie. Comme cela est magistralement montré, les Yanesha sont une véritable société végétaliste pour reprendre l'expression de l'ethnobotaniste du MNHN Jacques Barrau (1991) comme le précise J.-P. Chaumeil dans la préface de l'ouvrage.

2 L'auteure, en trois parties et huit chapitres, nous propose une réflexion sur la médecine yanesha en allant de la représentation de la personne et son positionnement au monde jusqu'à la mort et la vie dans l'au-delà. Chaque fin de chapitre comprend un utile récapitulatif de ce qui a été exposé.

3 Le chapitre premier traite de la physicalité en exposant la fabrication de l'être et en distinguant les différentes composantes du corps yanesha : le corps physique proprement dit (yénar), le principe vital (yecamquëm) et la joliment nommée ombre réprimée (yechoyeshe'm). Les Yanesha identifient leur tunique (cushma) comme un véritable supplément d'âme: « une partie intégrée et intégrante de l'être ». L'auteure dresse une 
analogie stimulante entre cette cushma, "élément de la personne yanesha qui signe l'appartenance à l'espèce humaine, mais aussi permet d'être, au sein de cette espèce, reconnu comme un sujet ", et les peintures corporelles qui jouent un rôle important dans la construction identitaire et l'ontologie de nombreux peuples amazoniens. D'ailleurs ces tuniques sont elles-mêmes ornées de motifs... S'amorce ici une très intéressante réflexion en phase avec les travaux contemporains sur le perspectivisme et l'ontologie des peuples amazoniens. Le chapitre se clôt sur l'explication de ce qu'est l'ombre errante (choyeshe'mats), source de bien des craintes pour les vivants. L'auteur nous signale que cette entité ne se nourrit que de produits carbonisés rappelant ainsi qu'au-delà du cru et du cuit, il y a le brûlé...

4 Le deuxième chapitre nous plonge dans l'étiologie yanesha et nous montre comment certaines entités sont source de dysfonctionnements corporels. L'auteure nous immerge ainsi dans le mode de pensée yanesha en montrant comment la haine, l'envie et la frustration sont à l'origine de nombreuses perturbations émotionnelles. En plus de nous décrire et d'expliquer le comportement et l'ontologie de ces entités, Valadeau nous les donne littéralement à voir grâce à de magnifiques dessins réalisés par un de ses collaborateurs yanesha, Anselmo Cruz Mariño. Les représentations graphiques d'entités subtiles, d'épisodes mythologiques ou de représentations cosmologiques par des Amérindiens constituent selon nous un indéniable apport aux travaux académiques. Plus qu'une simple illustration, ces dessins peuvent permettre de mieux appréhender la physicalité d'entités que l'on a souvent du mal à décrire. Il faut cependant être bien conscient de la part de subjectivité et de biais que cette démarche peut induire: les représentations graphiques à l'instar des mythologies sont en effet tout sauf immuables.

Le chapitre suivant nous explique l'origine des plantes médicinales (pare'shemats) ainsi que leur logique de nomination. Comme dans beaucoup d'autres mythologies amazoniennes, ces plantes ont comme origine la transformation des corps de divinités et d'ancêtres ou de leurs sécrétions corporelles (sang ou lait chez les Yanesha); parfois elles ont été données telles quelles par différentes entités. On apprend ainsi que la plante pa'yon (Tetragastris panamensis), qui sert à teindre la cushma yanesha d'ocre-rouge et dont la décoction d'écorce (rouge sang) est utilisée en cas d'hémorragie féminine, est la transformation du sang de Shopsheresyac, épouse de la divinité Yompor Oressem. Avec un tel pédigrée on ne s'étonnera pas de l'importance de ce vêtement dans la culture yanesha. Les plantes à haute valeur culturelle ont été données directement par des divinités, telles que ces plantes domestiquées que sont le tabac ou les fameux charmes magiques et médicinaux nommés piri piri ou souchet (Cyperus sp.), epe' en yanesha. Ces charmes (qui sont le plus souvent de chasse ou de séduction) sont particulièrement intéressants car s'ils existent chez un grand nombre de peuples amazoniens, ils appartiennent pour l'essentiel à deux familles botaniques bien différentes: les Cyperacées (genre Cyperus notamment) ou bien les Aracées (genres Caladium et Xanthosoma) (Grenand et al. 2004). Remarquons que l'usage de ces deux taxons botaniques correspond à de grandes zones géographiques. Ainsi sur le plateau des Guyanes les Aracées sont les principales plantes utilisées comme charmes, alors qu'au Pérou ou en Bolivie on se sert des Cypéracées. Il est intéressant de noter que là où la nomenclature vernaculaire reconnaît des dizaines de taxons bien différenciés avec un nom et des usages bien précis, les botanistes ont bien souvent du mal à les distinguer et les regroupent en quelques espèces. Une passionnante étude comparative sur les végétaux à charme à l'échelle pan-amazonienne reste à mener. D'ailleurs, tout récemment, Van Andel et al. (2015) ont écrit un article de synthèse fort utile sur les charmes amérindiens du plateau des Guyanes. Dans ce troisième chapitre, 
Valadeau nous montre que la classification vernaculaire de ces fameuses plantes se base sur des critères ontologiques. Et d'expliquer que «nommer une plante c'est répéter le processus ontologique ayant donné lieu à son apparition ».

6 La deuxième partie de l'ouvrage s'interroge sur « le choix d'une thérapeutique comme cause de la vulnérabilité de l'être ». Le chapitre IV montre comment la conception de la bonne santé chez les Yanesha est liée à la bonne adhérence au corps du principe vital yecamquëm : celui-ci doit être maintenu au plus près du corps. Ainsi certaines plantes permettent soit de maintenir cette bonne adhérence, soit de remplacer les pertes quotidiennes inhérentes à la corporalité yanesha. Celles-ci sont constituées des excrétions de différents fluides corporels (sperme, sang, sueur, lait...); ainsi " pour un adulte, se maintenir en bonne santé consiste, tout d'abord, à remédier à ces pertes quotidiennes de principe vital dues à des productions corporelles, considérées comme normales, physiologiques ». Ensuite, par un enchaînement logique, Valadeau nous donne à comprendre la conception yanesha de la maladie en nous montrant les mécanismes pathologiques influençant directement le principe vital. Les frayeurs subites, la rencontre avec les ombres errantes ou bien les pertes extra-somatiques peuvent ainsi entrâner le décollement ou l'arrachement d'une partie du principe vital. Mais un surplus de celui-ci peut également être source de mauvaise santé. Deux grands types de douleurs sont reconnus par les Yanesha: les douleurs internes et les douleurs externes ou périphériques (cutanées). L’importance donnée aux affections cutanées se rencontre chez de nombreux peuples amazoniens (comme chez les Wayana, peuple karib de Guyane française). Ici, l'auteure montre le rôle central des entités diurnes associées à l'arc-en-ciel et des entités nocturnes issues des milieux humides.

7 Nous n'en dirons pas plus et invitons le lecteur à découvrir par lui-même ce livre enthousiasmant. Il nous semble important de mentionner que rares sont les travaux de ce type couplant une analyse fine d'anthropologie médicale à une botanique riche et précise. Car, même si nous aurions aimé que l'ouvrage comprenne un index final faisant correspondre les taxons yanesha avec leur détermination botanique, il n'en reste pas moins que cette recherche captivera toutes les personnes intéressées par l'ethnobotanique, l'ethnomédecine et plus largement tous ceux que les savoirs liés à la nature passionnent.

\section{BIBLIOGRAPHIE}

ANDEL Tinde van, Sofie RUYSSCHAERT, Karin BOVEN et Lewis DALY

2015, "The use of Amerindian charm plants in the Guianas ", Journal of ethnobiology and ethnomedicine [en ligne], 15 September 2015, https://ethnobiomed.biomedcentral.com/articles/ 10.1186/s13002-015-0048-9 (DOI 10.1186/s13002-015-0048-9), consulté le 31 août 2016.

GrenAND Pierre, Christian MORETTI, Henri JACQUEMIN et Marie-Françoise PRÉVOST 2004, Pharmacopées traditionnelles en Guyane. Créoles, Wayãpi, Palikur, IRD Éditions, Paris. 
AUTEURS

DAMIEN DAVY

UMSR LEEISA (CNRS-UG-IFREMER), Cayenne 